\title{
A Novel Ameliorated Slotted ALOHA Protocol
}

\author{
Wang Yiming \\ The First Institute of Oceanography \\ SOA. \\ Qingdao, China \\ edit231@163.com
}

\begin{abstract}
Aloha or later slotted Aloha is known as the first protocol for packet radio network and a classic example of random access protocol. However, their performance is not promising as they can only achieve maximum throughput of $18 \%$ and $36 \%$ for Aloha and slotted Aloha, respectively. Moreover it will be unstable for increasing traffic. An improvement protocol to raise the performance of slotted Aloha, based on slot reservation for managing collision in slotted Aloha system is proposed. Using this method, the chance of a re-collision for retransmitted packets is minimized. This paper studied the performance of Aloha, slotted Aloha and the proposed improved protocol using smpl simulation library to simulate the system. The results show that the simulation designed for Aloha and Slotted Aloha conform to the theoretical data. It also shows that the protocol improvement can raise the performance of Slotted aloha about $3.5 \%$. Analysis is provided for each simulation data.
\end{abstract}

Keywords-Access protocols, Collision processes, Multi-access communication, Protocols

\section{INTRODUCTION}

In recent years, the ALOHA protocal has extensively been studied. Considerable progress has been made in the field of non-deterministic distributed random access protocols since ALOHA was introduced by Abramson. However, due to its simplicity and classic, ALOHA and later slotted ALOHA are still in use for academic research. ALOHA protocol suggests that any transmitter nodes that have data will transmit immediately without delay. If there are collisions, retransmission performed. To reduce the frequent retransmission, vulnerable period is set to two times of the packets transmission time needed. This restrains the normalized throughput to maximum $18 \%$ [1-9].

The slotted system idea doubles the maximum throughput in the sense that it avoids the collision between the ongoing packets and packets arriving after. The drawback is that synchronized transmissions to time slots wait for beginning of the fixed time interval and transmit with certainty. The chance of collision is high. The next major step towards high capacity random access is carrier sensing. However, for channels with moderate or long propagation delay, its performance is not that excellence. It becomes especially obvious when the data packet is very small. In the situation of limited hardware resources and low data rate communication or as a component in many

\author{
Yang Guang, Xing Youguang \\ Shandong Provincial Key Laboratory of Ocean \\ Environment Monitoring Technology \\ Shandong Academy of Sciences Institute of \\ Oceanographic Instrumentation \\ Qingdao, China
}

complicated reservation protocols concerned, ALOHA protocol is still beneficial.

This paper intends to explore the performance of the slotted ALOHA using an event-driven SMPL simulator. The diversity between the experimental result and theoretical result will be interpreted. In this paper, a new improvement aimed to increase the overall throughput of the slotted ALOHA protocol will be introduced.

\section{COllision Avoidance Algorithm}

The algorithms described in this section are based on the following assumptions regarding to the channel scenario:

(a) All transmitted messages are of fixed length, $\mathrm{T}$ seconds, as in slotted ALOHA systems.

(b) Terminals transmitting a packet on the channel receive feedback about channel events with a known deterministic delay of zero seconds in the end of transmission.

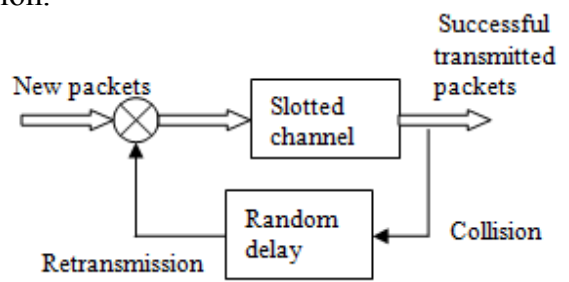

Figure 1. Slotted ALOHA

Referring to Figure 1, slotted random access protocols can be specified in terms of both a new transmission and a retransmission procedure. Both procedures are the simplest possible, that is transmit new messages as soon as they are generated (new transmission rule) and retransmit after a random delay (retransmission rule) with constraint that all transmissions begin only on slot boundaries.

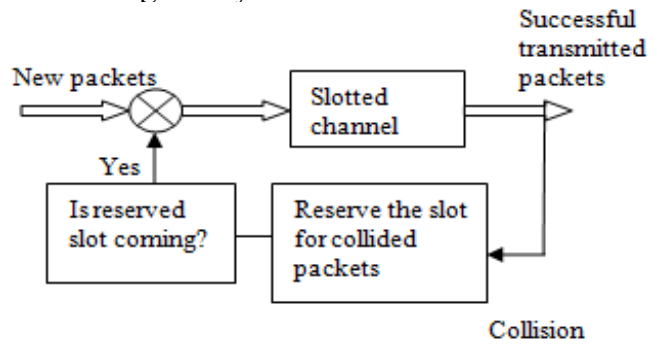

Figure 2. Slotted ALOHA with CRA 
The retransmission slot reservation collision resolution algorithm proposed here, shown schematically in Figure2, are characterized by two channel modes: a synchronous new packets transmission mode and a locally synchronous collision resolution retransmission mode. The new packets transmission mode is no difference with the traditional slotted ALOHA algorithm.

The second mode differs with the traditional slotted ALOHA. To see the improvement in the new algorithm, a case of packets collision is studied:

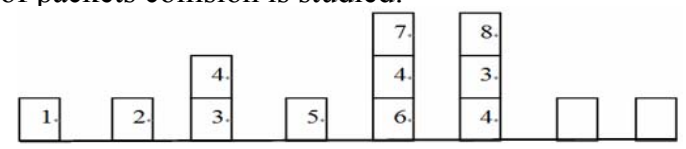

Figure 3. Illustration of slotted ALOHA

Referring to Figure3, packet 3 collides with packet 4 in a slotted ALOHA system. After a random delay, packet 4 retransmits; unfortunately it collides again with packet 6 and 7. The retransmitted packet 3 and 4 collides with the new packets 8 in the next time slot. The throughput is kept down by the repeating collision involving the retransmitted packets.

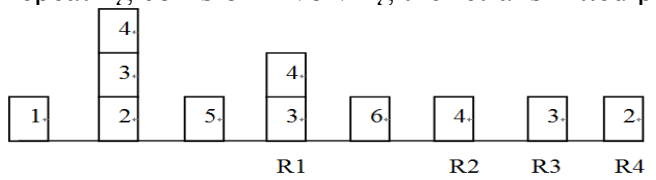

Figure 4. Illustration of slotted ALOHA with slot reservation

Note: R1, R2, R3 represent slots reserved for retransmissions

Referring to Figure4, packet 2, 3 and 4 collide, slots R1, $\mathrm{R} 2, \mathrm{R} 3$ are reserved for their retransmissions. In R1, although the chance of three packets collides is low, it is possible retransmission packet 3 and 4 are collided. In this situation, another reserved slot R4 is created. Upon successfully retransmitted packet 4 , the chance of collision happened to the other 2 retransmission packets are further reduced. It is obvious that the suggested collision avoidance algorithm improves the ALOHA protocol. This is done by avoiding the possibility that a packet which has already been subjected to a collision encounters another new packet during its retransmission. The new packets are not allowed to transmit in time slots provided for retransmission; additional access control is to prevent conflicts with retransmissions.

The random selection of the reservation slots will reduce the delays caused by the reservation scheme. Throughput is increased at the expense of complexity in reserving the slots and probably a short delay in the new transmitting packets for retransmissions. The following part describes the actual implementation of the simulation algorithms.

\section{SimUlation DATA AND ANALYSIS}

The simulation program is run with parameters set for varying number of transmitters listed in Table I.
TABLE I. SIMULATION PARAMETER SET

\begin{tabular}{|l|l|}
\hline Parameter & value \\
\hline Transmission time & 5 cycle time \\
\hline Interval of arrival time & $\begin{array}{l}\text { exponentially distributed } \\
\text { with mean of: } 50 \text { cycle times }\end{array}$ \\
\hline $\begin{array}{c}\text { Observation period for } \\
\text { each simulation: }\end{array}$ & $10^{\wedge} 6$ cycle time \\
\hline Number of transmitters & 1 to 50 \\
\hline
\end{tabular}

\section{A. Normalized throughput with varying Offered Load}

Below shows the throughput performance of Aloha, Slotted Aloha and the retransmission slot reservation scheme with respect to offered load.

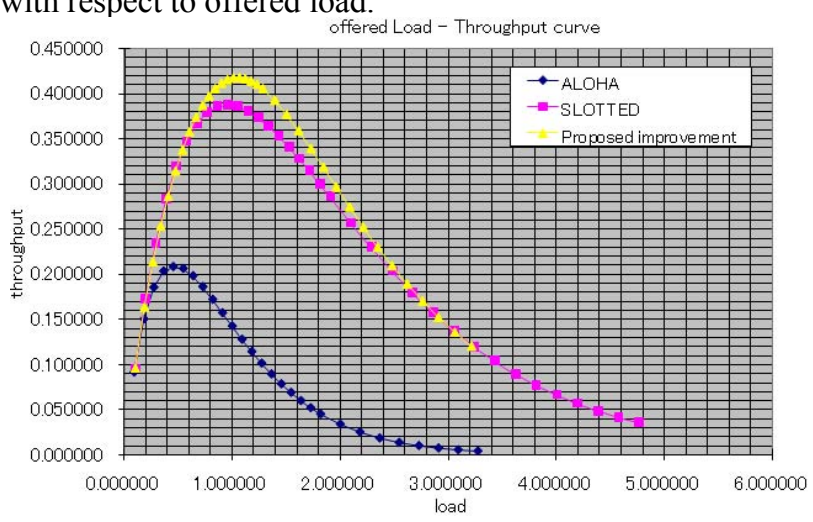

Figure 5. Simulations on Throughput VS Offered load of ALOHA, slotted ALOHA and Improved algorithm

For Aloha, the maximum throughput was 20.5\%, achieved when offered load is: 0.54 . For Slotted Aloha, the maximum throughput is: $38.6 \%$ achieved when offered load was 0.95 . The result above conform with well-known Aloha and Slotted aloha performance where maximum throughput is $18.4 \%$ (when offered load: 0.5 ) and $36.8 \%$ (when offered load: 1) respectively. From throughput graphs, it can also be inferred that number of successful transmission will be higher in slotted aloha. As discussed, In Slotted Aloha, two packets either collides completely or doesn't overlap at all since it have time slot to transmit. Hence, Slotted Aloha will have less vulnerable period, and accordingly, the probability of successful transmission is higher than pure Aloha with $\mathrm{P}$ (successful transmission) $=e^{-\tilde{G}}$. From the simulation, it is shown both Aloha and Slotted-Aloha will not be stable with increasing load. The throughput will drop after it reach its optimum load (0.54 and 0.95 for Aloha and Slotted aloha, respectively), and approaching 0 for larger load. It can be analyzed as following:

Let's take example for Slotted- Aloha system with $\tilde{S}=\tilde{G} x e^{-\tilde{G}}$. Probability of successful transmission $\left(1 / e^{\tilde{G}}\right)$ will drop with increasing $\tilde{G}$, and after some point, the dropping rate is getting higher than increasing rate of $\tilde{G}$. Thus, from this point on, we will see that the successful transmission (i.e. throughput) will also drop. We may further observe when load reach infinity: 


$$
\begin{aligned}
& \lim _{\tilde{G} \rightarrow \infty} \tilde{S}(\text { slotted })=\lim _{\tilde{G} \rightarrow \infty} \frac{\tilde{G}}{e^{\tilde{G}}} \\
& \text { Applying: } e^{x}=1+x+\frac{x^{2}}{2 !}+\frac{x^{3}}{3 ! !}+\ldots, \text { we get : } \\
& \lim _{\tilde{G} \rightarrow \infty} \tilde{S}(\text { slotted })=\lim _{\tilde{G} \rightarrow \infty} \frac{\tilde{G}}{1+\tilde{G}+\frac{\tilde{G}^{2}}{2 !}+\frac{\tilde{G}^{3}}{3 !}+\ldots} \times \frac{1 / \tilde{G}}{1 / \tilde{G}} \\
& \lim _{\tilde{G} \rightarrow \infty} \tilde{S}(\text { slotted })=\lim _{\tilde{G} \rightarrow \infty} \frac{1}{\frac{1}{\tilde{G}} /+1+\frac{\tilde{G}}{2 !}+\frac{\tilde{G}^{2}}{3 !}+\ldots}=0
\end{aligned}
$$

Thus, it is proved that Slotted Aloha will not support increasing load as it will give 0 throughputs when Load approaches infinity. Similar observation with similar result can be done for Aloha. For the retransmission slot reservation scheme it can be shown in the simulated plot that the maximum throughput is increased to about $42 \%$ compared to $38.6 \%$ from the slotted ALOHA. This is achieved by reducing the chance of collision between the retransmitted packets and new transmission packets. However, when the retransmissions become more frequent, the chance of collision in retransmitted packet increase dramatically. The advantage of retransmission slot reservation becomes not obvious when the offered load reached around 3.

\section{B. Normalized throughput with varying number of transmitters}

This section study the performance of the Aloha, slotted Aloha and the retransmission slot reservation scheme from the perspective of how it can support varying number of transmitters. From the simulation, the throughput with varying number of transmitters is plotted as below:

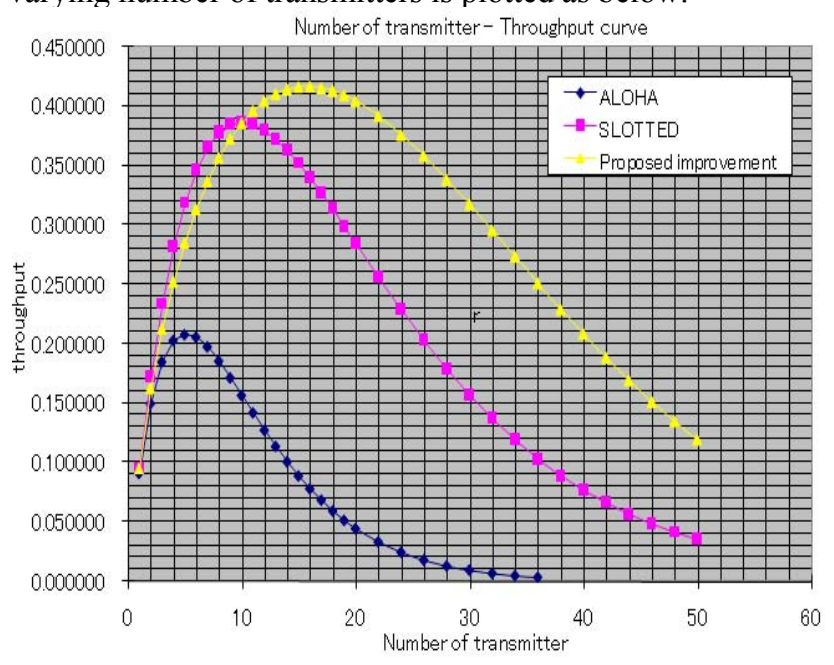

Figure 6. Simulations on Throughput VS Number of Transmitter of ALOHA, slotted ALOHA and Improved algorithm

From the observation of Figure6, the maximum throughput of the improved algorithm reaches about $42 \%$. It is worth mentioning that the throughput drop is not that rapid. In the case of 30 transmitters, throughput of slotted ALOHA dropped to $16 \%$, while slot reservation scheme, it keeps high at $32 \%$. It can be seen that the throughput vs. offered load and throughput vs. number of transmitters shows different results for the retransmission slot reservation scheme.

\section{CONCLUSION}

In this paper, we have presented a thorough analysis and comparison of the Aloha, slotted Aloha and the proposed retransmission slot reservation scheme. The analysis shows that not only the proposed protocol improvement increase the maximum throughput by approximately $3.5 \%$ compared to slotted Aloha; it also reduces the offered load by decreasing the amount of retransmissions via a more efficient retransmission rule. We have also shown that by adjusting the reservation interval sizes, we are able to reduce the rate of degradation of the throughput significantly when the number of transmitters increases.

\section{REFERENCES}

[1] S. Ghez, S. Verdu, and S. C. Schwartz, "Stability properties of slotted ALOHA with multipacket reception capability,” IEEE Trans. Autom. Control, vol. 33, no. 7, pp. 640-649, Jul. 1988.

[2] W. Szpankowski, "Stability conditions for some distributed systems: Buffered random access systems," Adv. Appl. Probabil., vol. 26, pp. 498-515, 1994.

[3] B. Tsybakov and V. Mikhailov, "Ergodicity of slotted ALOHA system," Problemy Peredachi Informatsii, vol. 15, pp. 73-87, Oct.Dec. 1979.

[4] R. Rao and A. Ephremides, "On the stability of interacting queues in a multiple-access system," IEEE Trans. Inf. Theory, vol. 34, pp. 918930, Sep. 1988.

[5] W. Luo and A. Ephremides, "Stability of $\mathrm{n}$ interacting queues in random access systems," IEEE Trans. Inf. Theory, vol. 45, pp. 15791587, Jul. 1999.

[6] M. Zorzi and R. Rao, "Capture and retransmission in mobile radio," IEEE J. Sel. Areas Commun., vol. 12, no. 8, pp. 1289-1298, Oct. 1994.

[7] B. Hajek, A. Krishna, and R. LaMaire, "On the capture probability for a large number of stations," IEEE Trans. Commun., vol. 45, no. 2, pp. 254-260, Feb. 1997.

[8] V. Naware, G. Mergen, and L. Tong, "Stability and delay of finiteuser slotted aloha with multipacket reception," IEEE Trans. Inf. Theory, vol. 51, no. 7, pp. 2636-2656, 2005.

[9] Minh Hanh Ngo. Optimal Channel-Aware ALOHA Protocol for Random Access in WLANs with Multipacket Reception and Decentralized Channel State Information, IEEE TRANSACTIONS ON SIGNAL PROCESSING, VOL. 56, NO. 6, JUNE, 2008, pp.2575-2588. 